\title{
A Note on Non-Negative Singular Infinity-Harmonic Functions in the Half-Space
}

\author{
Tilak BHATTACHARYA \\ Department of Mathematics \\ Purdue University \\ W. Lafayette, IN 47907 - USA \\ tbhatta@math.purdue.edu
}

Recibido: 5 de enero de 2005

Aceptado: 4 de abril de 2005

\begin{abstract}
In this work we study non-negative singular infinity-harmonic functions in the half-space. We assume that solutions blow-up at the origin while vanishing at infinity and on a hyperplane. We show that blow-up rate is of the order $|x|^{-1 / 3}$.
\end{abstract}

Key words: viscosity solutions, infinity-harmonic, singular.

2000 Mathematics Subject Classification: 35J70, 36A16.

\section{Introduction.}

Our effort in this note will be to derive growth rates for non-negative singular infinityharmonic functions in the half-space. The functions of interest will have a singularity at the boundary while vanishing elsewhere on the hyperplane and at infinity. In particular we will show that any two such singular functions are comparable and thus have the same growth rate. This is to be viewed as a follow-up of [7] where singular infinity-harmonic functions were studied in greater generality. In the present case the precise nature of the growth rate will follow by adapting an example constructed in [2]. Our framework in this note will be that of viscosity solutions and to describe our results more precisely, we introduce the following notations. Let $x=\left(x_{1}, x_{2}, \ldots, x_{n}\right)$ denote a point in $\mathbb{R}^{n}, n \geq 2, O=(0,0, \ldots, 0)$ be the origin and $H=\left\{x: x_{n}>0\right\}$

A part of the work was done while the author was being supported by a fund from the NSERC. 
the half-space. We define $u=u(x)$ to be infinity-harmonic (or $\infty$-harmonic) in $H$, if $u$ solves

$$
\Delta_{\infty} u(x)=\sum_{i, j=1}^{n} \frac{\partial u}{\partial x_{i}} \frac{\partial u}{\partial x_{j}} \frac{\partial^{2} u}{\partial x_{i} \partial x_{j}}=0, \quad \forall x \in H,
$$

in the viscosity sense $[3,6,9,10]$. It is well known that $u$ is locally Lipschitz continuous and obeys the comparison principle. Let $B_{R}(P)$ be the open ball in $\mathbb{R}^{n}$ with center $P$ and radius $R, B^{+}(R, O)=B(R, O) \cap H$, and $S_{R}=\partial B(R, O) \cap H$. Also let $T=\left\{x: x_{n}=0\right\}$. Set $M(R)=\sup _{S_{R}} u(x), R>0$. We will always assume that $u(x)>0$, for every $x \in H$ and

(A1) $u(x)$ is continuous up to $T \backslash\{O\}$ and $u(x)=0, x \in T \backslash\{O\}$,

(A2) for every $R>0,0<M(R)<\infty, \sup _{R>0} M(R)=\infty$, and $\lim _{R \rightarrow \infty} M(R)=0$.

Note that in (A2) we do not specify any growth or decay rates and the blow-up occurs only at the origin $O$. Our main result in this work is

Theorem 1.1 (Comparison). Let $u>0$ and $v>0$ be infinity-harmonic in $H$ and satisfy the assumptions (A1) and (A2). For every $R>0$, let

$$
S(R)=\sup _{x \in \partial B_{R}(O) \cap H} u(x) / v(x) \quad \text { and } \quad s(R)=\inf _{x \in \partial B_{R}(O) \cap H} u(x) / v(x) .
$$

Then

(i) $S(0)=\lim _{R \rightarrow 0} S(R)<\infty$ and $\lim _{R \rightarrow 0} s(R)=s(0)>0$ exist,

(ii) there exists a universal constant $C>0$ such that $S(0) \leq C s(0)$,

(iii) for every $x \in H, \frac{S(0)}{C} \leq \frac{u(x)}{v(x)} \leq S(0)$.

Moreover, any non-negative singular solution is axially symmetric, i.e., $u(x)=h(r, \theta)$, where $r=r(x)=|x|$ and $\theta=\theta(x)=\cos ^{-1} x_{n} / r$.

In light of the example in [2] and the discussion in the Appendix, it follows that $u(x) \sim|x|^{-1 / 3}$. This result then improves the lower bound proven in [7] in this special case (see [7, Remark 3]). The main ingredients of the proof are the Harnack inequality $[3,5,6,11]$, the boundary Harnack principle [6] and the comparison principle [3,4]. The question as to whether $S(0)=s(0)$ is true, thereby showing that $u(x)=C v(x)$, is unclear to us. If true it would then show that such solutions are separable in $r$ and $\theta$. In section 2, we first prove a somewhat sharper version of the Harnack inequality which will provide a lower bound for a solution $u$. We then present the proofs of parts (i)-(iii) of Theorem 1.1. This will then be followed up by the proof of the fact that $u(x)$ has axial symmetry with respect to the $x_{n}$-axis. Additional properties will also be shown. In the Appendix we recall the example studied in $[1,2,7]$. Finally, we remark that if a boundary Harnack inequality could be proven for $C^{2}$ domains then a version of Theorem 1.1 would hold for cones. 


\section{Proofs of Theorem 1.1 and other results.}

We first state a more refined version of the Harnack inequality. See $[3,6,11]$.

Lemma 2.1 (Harnack's inequality). Let $\Omega \subset \mathbb{R}^{n}$, be an open and connected set. Let $u>0$ be infinity-harmonic in $\Omega$. Suppose $A$ and $B$ are two points in $\Omega$ and $\sigma(t), 0 \leq t \leq 1$, be a smooth curve from $A$ to $B$ with $\sigma(0)=A$ and $\sigma(1)=B$. Let $\delta(t)=\operatorname{dist}(\sigma(t), \partial \Omega) \neq 0$, then

$$
u(A) \leq u(B) \exp \left(\int_{0}^{1} \frac{\left|\sigma^{\prime}(t)\right|}{\delta(t)} d t\right) .
$$

Proof. First recall that if $P_{1}$ and $P_{2}$ are any two points in $\Omega$ that are joined by a straight segment that is at least $\eta$ away from $\partial \Omega$, then $u\left(P_{1}\right) \leq u\left(P_{2}\right) e^{\left|P_{1}-P_{2}\right| / \eta}$ $[3,5-7]$. Now partition $[0,1]$ and approximate the curve by finitely many chords. Since the Harnack inequality is multiplicative, the finite sum may then be replaced by a Riemann integral by successive refinement of the partition.

We now prove a comparison result which will be applied in what follows.

Lemma 2.2 (Comparison). Let $u_{1}>0$ and $u_{2}>0$ be two infinity-harmonic functions in $H \backslash B_{R}(O), R>0$. For $i=1,2$, assume that

(i) $\lim _{r \rightarrow \infty} \sup _{\{|x|=r\} \cap H} u_{i}(x)=0$,

(ii) $u_{i}(x)$ is continuous up to $\left(T \backslash B_{R}(O)\right) \cup\left(\partial B_{R}(O) \cap H\right)$, and

(iii) $u_{i}(x)=0, x \in T \backslash B_{R}(O)$.

If $u_{1}(x) \leq u_{2}(x), x \in \partial B_{R}(O) \cap H$, then $u_{1}(x) \leq u_{2}(x), x \in H \backslash B_{R}(O)$.

Proof. Let $\varepsilon>0$ be arbitrary and $r_{\varepsilon}$ be such that $u_{1}(x) \leq \varepsilon$, for every $x \in H \backslash B_{r_{\varepsilon}}(O)$. For $r>r_{\varepsilon}$, consider the region $P_{r, \varepsilon}=\{x \in H: R<|x|<r\}$. Let $u_{2, \varepsilon}(x)=u_{2}(x)+\varepsilon$. Then

(i) $u_{2, \varepsilon}(x)$ is $\infty$-harmonic in $P_{r, \varepsilon}$,

(ii) $u_{2, \varepsilon}(x) \geq u_{1}(x), x \in\left(\partial B_{r}(O) \cup \partial B_{R}(O)\right) \cap H$, and

(iii) $u_{2, \varepsilon}(x) \geq u_{1}(x), x \in T \cap\left(B_{r}(O) \backslash B_{R}(O)\right)$.

By comparison $u_{1}(x) \leq u_{2, \varepsilon}(x), x \in P_{r, \varepsilon}$. Since $r$ and $\varepsilon$ are arbitrary $u_{1}(x) \leq u_{2}(x)$, $x \in H \backslash B_{R}(O)$.

Corollary 2.3. For $i=1,2$, let $u_{i}>0$, be infinity-harmonic in $H \backslash B_{R}(O)$ and satisfy the assumptions (i)-(iii) in Lemma 2.2. For every $r \geq R$, define $S(r)=$ $\sup _{\{|x|=r\} \cap H} u_{1}(x) / u_{2}(x)$ (possibly infinite) and $s(r)=\inf _{\{|x|=r\} \cap H} u_{1}(x) / u_{2}(x)$ (possibly zero). Suppose that $S(\rho)<\infty$ for some $\rho \geq R$, then $S(r)$ is decreasing in $r$ for $r \geq \rho$. Analogously if $s(\rho)>0$ then $s(r)$ is increasing in $r$ when $r \geq \rho$. 
Proof. We use comparison. Let $w(x)=S(\rho) u_{2}(x)$. Clearly $w(x) \geq u_{1}(x)$, for every $x \in \partial B_{\rho}(O) \cap H$. Clearly, the assumptions (i)-(iii) in Lemma 2.2 are then met and $S(\rho) u_{2}(x) \geq u_{1}(x)$, for every $x \in H \backslash B_{\rho}(O)$. Thus for every $r \geq \rho$ it follows that $S(r)=\sup _{\{|x|=r\} \cap H} u_{1}(x) / u_{2}(x) \leq S(\rho)$. Replacing $\rho$ by $r$ and applying the above argument the conclusion follows. To obtain the conclusion for $s(r)$ we work with $w(x)=s(\rho) u_{2}(x)$.

The basic argument used in Lemma 2.2 will be often used in the rest of this work. Our proof of Theorem 1.1 will involve the application of the boundary Harnack inequality and Corollary 2.3. For notational ease we will often write $x=$ $\left(x_{1}, x_{2}, \ldots, x_{n}\right)=\left(x^{\prime}, x_{n}\right)$. For $Q=\left(Q^{\prime}, 0\right) \in T$, let $(Q, d)$ denote the point $\left(Q^{\prime}, d\right)$ and $(0, d)$ the point on $x_{n}$-axis with $x_{n}=d$. Also $|x|^{2}=\sum_{i=1}^{n} x_{i}^{2}$ and $\left|x^{\prime}\right|^{2}=\sum_{i=1}^{n-1} x_{i}^{2}$.

Proofs of parts (i)-(iii) of Theorem 1.1. Let $u(x)>0$ and $v(x)>0$ be two singular solutions in $H$ satisfying the assumptions (A1) and (A2). We will use the boundary Harnack principle [6] near the flat boundary $T$. For every $R>0$, let $J(R)=$ $\partial B_{R}(O) \cap T$, and for any $P \in J(R)$ set $D(P, R)=\{x \in T:|x-P|<R\}$. Let $C(P, R)=D(P, R) \times(0,2 R)=\left\{x \in H:\left(x^{\prime}, 0\right) \in D(P, R), 0<x_{n}<2 R\right\}$ denote the cylinder of height $2 R$, radius $R$, with axis parallel to $x_{n}$-axis and $P$ the center of the flat face on $T$. Fix $R>0$. By the boundary Harnack principle, there exist absolute constants $C_{1}$ and $C_{2}$ such that

$$
C_{1} \frac{u(P, R / 4)}{v(P, R / 4)} \leq \frac{u(x)}{v(x)} \leq C_{2} \frac{u(P, R / 4)}{v(P, R / 4)}, \quad \forall x \in C(P, R / 8) .
$$

We now relate $u(P, R / 4) / v(P, R / 4)$ to $u(0, R) / v(0, R)$ by using the regular Harnack inequality in Lemma 2.1. Let $L$ be the segment joining $(0, R)$ to $(P, R / 4)$, then $\operatorname{dist}(L, H) \geq R / 4$. Thus $e^{-5} u(0, R) \leq u(P, R / 4) \leq e^{5} u(0, R)$. With new absolute constants $C_{3}$ and $C_{4}$, (1) yields

$$
C_{3} \frac{u(0, R)}{v(0, R)} \leq \frac{u(x)}{v(x)} \leq C_{4} \frac{u(0, R)}{v(0, R)}, \quad \forall x \in C(P, R / 8) .
$$

By taking the union over all $P \in J(R)$, (2) holds for $x \in \partial B_{R}(O) \cap\left\{x: 0<x_{n} \leq\right.$ $\left.\left|x^{\prime}\right| / 4\right\}$. Using the Harnack inequality, we now show that an analogous estimate holds for $x \in \partial B_{R}(O) \cap\left\{x: x_{n} \geq\left|x^{\prime}\right| / 4\right\}$. If $y \in \partial B_{R}(O) \cap\left\{x: x_{n} \geq\left|x^{\prime}\right| / 4\right\}$ then $y_{n} \geq R / \sqrt{17}$ and $\left|y^{\prime}\right| \leq 4 R / \sqrt{17}$. The Harnack inequality then implies that $e^{-7} u(y) \leq u(0, R) \leq e^{7} u(y)$. This together with (2) implies that there are absolute constants $C_{5}$ and $C_{6}$ such that for every $R>0$ and $x \in \partial B_{R}(O)$,

$$
C_{5} \frac{u(x)}{v(x)} \leq \frac{u(0, R)}{v(0, R)} \leq C_{6} \frac{u(x)}{v(x)}, \quad \forall x \in \partial B_{R}(O) \cap H .
$$

For every $R>0$, it is clear that $0<s(R) \leq S(R)<\infty$, and there is an absolute constant $C_{7}$ such that $S(R) \leq C_{7} s(R)$. By Corollary 2.3, $S(R)$ is decreasing in $R$ and 
$s(R)$ is increasing in $R$. Taking limits we have that $S(0) \leq C_{7} s(0)$. By Lemma 2.2, for every $R>0, s(0) \leq s(R) \leq u(x) / v(x) \leq S(R) \leq S(0), x \in H \backslash B_{R}(O)$. Thus parts (i)-(iii) of Theorem 1.1 are now proven.

The rest of this section is devoted to deriving additional properties of non-negative singular solutions including the last conclusion in Theorem 1.1 (see Lemma 2.6). For $i=1,2, \ldots, n$, let $\overrightarrow{e_{i}}$ be the unit vector along the $x_{i}$-axis.

Remark 2.4. Recall that $M(r)=\sup _{\partial B_{r}(O) \cap H} u(x)$. A comparison argument involving the function $w(x)=M(a)+\frac{M(b)-M(a)}{b-a}(|x|-a), x \in H, a \leq|x| \leq b$, shows that $M(r)$ is convex in $r$. Since $M(r)>0$ and $u$ satisfies (A2), it follows that $M(r)$ is decreasing in $r$.

Lemma 2.5. Let $u$ be as in Theorem 1.1, and for $r>0$ let $M(r)$ be as defined above. Let $D_{r}(O)$ be a fixed great circle centered at $O$. For every $x \in D_{r}(O)$ define $\theta=\theta(x)=\cos ^{-1} x_{n} / r$. Then $u(x)$ is decreasing in $\theta$ and $u(0, r)=M(r)$.

Proof. We give a proof based on reflection and comparison. Fix $r>0$, and let $A(r)$ be the point $(0, r)$ on the $x_{n}$-axis. Let $\vec{\eta}=\left(\eta_{1}, \eta_{2}, \ldots, \eta_{n-1}, 0\right)$ be a unit vector orthogonal to the $x_{n}$-axis, and $\Pi(\vec{\eta})$ be the 2-dimensional plane containing the $x_{n^{-}}$ axis and $\vec{\eta}$. Select $\vec{\eta}$ such that $D_{r}(O)=\partial B_{r}(O) \cap \Pi(\vec{\eta})$. Let $P_{1}, P_{2} \in \partial D_{r}(O)$ (on one side of $x_{n}$-axis) be such that $0<\cos \theta_{1}=\frac{\left\langle P_{1}, e_{n}\right\rangle}{r}<\cos \theta_{2}=\frac{\left\langle P_{2}, e_{n}\right\rangle}{r} \leq 1$, where $\langle\cdot, \cdot\rangle$ denotes the inner product, and $\theta_{1}$ and $\theta_{2}\left(\theta_{1}^{r}>\theta_{2}\right)$ are the angles made with the $x_{n}$ axis. We show that $u\left(P_{1}\right) \leq u\left(P_{2}\right)$. This would imply that $u(x)$ increases along a great circle as $x \rightarrow A(r)$ (or as $\theta \rightarrow 0$ ). Let $\vec{e}=\left(P_{1}-P_{2}\right) /\left|P_{1}-P_{2}\right|$, then $-1<\left\langle\vec{e}, \overrightarrow{e_{n}}\right\rangle<0$. Set $P_{3}=\left(P_{1}+P_{2}\right) / 2$, and note $\vec{e} \perp \overrightarrow{P_{3}}$. Let

(i) $Z(\vec{e})=\left\{x:\left\langle x-P_{3}, \vec{e}\right\rangle=0\right\}$ be the hyperplane containing $O$ and $P_{3}$,

(ii) $Z(\vec{e})^{+}=\left\{x:\left\langle x-P_{3}, \vec{e}\right\rangle>0\right\}$ and $Z(\vec{e})^{-}=\left\{x:\left\langle x-P_{3}, \vec{e}\right\rangle<0\right\}$, the half-spaces,

(iii) $T(\vec{e})=T \cap Z(\vec{e})^{+}$, and

(iv) the infinite wedge $W(\vec{e})=H \cap Z(\vec{e})^{+}$.

For $x \in W(\vec{e})$, define the reflection about $Z(\vec{e})$ by $x^{f}=x-2\langle x, \vec{e}\rangle e$. Clearly $\left(x^{f}\right)^{f}=x$. Also set

(v) $W(\vec{e})^{f}=\left\{x: x^{f} \in W(\vec{e})\right\}$, the reflection of $W(\vec{e})$ about $Z(\vec{e})$, and

(vi) $T^{f}(\vec{e})$ the reflection of $T(\vec{e})$ about $Z(\vec{e})$.

Define $u^{f}(x)=u\left(x^{f}\right), x \in W^{f}(\vec{e})$, then $u^{f}$ is $\infty$-harmonic in $W^{f}(\vec{e})$. Noting that $u(x)>0$ in $W^{f}(\vec{e})$, comparison would then yield $u(x) \geq u^{f}(x)$ in $W^{f}(\vec{e})$. However, due to the singularity at $O$, we modify the geometry. For $\delta>0$, small, let $Z(\vec{e})_{\delta}=$ $\{x+\delta \vec{e}: x \in Z(\vec{e})\}=\left\{x:\left\langle x-P_{3}, \vec{e}\right\rangle=\delta\right\}$ be the translated plane and the half-spaces $Z(\vec{e})_{\delta}^{ \pm}$similarly defined. Set $W(\vec{e})_{\delta}=H \cap Z(\vec{e})_{\delta}^{+}$and let $W^{f}(\vec{e})_{\delta}$ be the reflection of 
$W(\vec{e})_{\delta}$ about $Z(\vec{e})_{\delta}$. Also for $x \in W(\vec{e})_{\delta}$, let $x_{\delta}^{f}=x+2(\delta-\langle x, \vec{e}\rangle) \vec{e}$. Also set $T_{\delta}^{f}(\vec{e})$ to be the reflection of $T \cap Z(\vec{e})_{\delta}^{+}$. Define $u_{\delta}^{f}(x)=u\left(x_{\delta}^{f}\right)$ in $W^{f}(\vec{e})_{\delta}$. Then

(a) $u_{\delta}^{f}(x)$ is $\infty$-harmonic in $W^{f}(\vec{e})_{\delta}$,

(b) $u_{\delta}^{f}(x)=u(x)$, on $Z(\vec{e})_{\delta} \cap H$, and

(c) $u(x) \geq u_{\delta}^{f}(x)=0$ in $T_{\delta}^{f}(\vec{e})$.

Adapting the argument of Lemma 2.2 we conclude that $u_{\delta}^{f}(x) \leq u(x), x \in W^{f}(\vec{e})_{\delta}$. Define $P_{2, \delta} \in W^{f}(\vec{e})_{\delta}$ the reflection of $P_{1}$. Clearly, $u\left(P_{1}\right)=u_{\delta}^{f}\left(P_{2, \delta}\right) \leq u\left(P_{2, \delta}\right)$. Since this holds for all small $\delta>0$, continuity of $u$ implies that $u\left(P_{1}\right)=u^{f}\left(P_{2}\right) \leq u\left(P_{2}\right)$. Clearly, the statement of the Lemma holds and $M(r)=u(0, r)$. Also by Remark 2.4, $u(0, r)$ is decreasing in $r$.

For $\theta \in[0, \pi / 2)$, let $C_{\theta}=\left\{x \in H: \cos ^{-1}\left(\frac{\left\langle x, e_{n}\right\rangle}{|x|}\right)=\theta\right\}$ be the cone of opening $\theta$ with apex at $O$ and symmetric about the $x_{n}$-axis. For $r>0$, let $I(\theta, r)=C_{\theta} \cap \partial B_{r}(O)$.

Lemma 2.6 (Symmetry). Let $u$ be as in Theorem 1.1. Then for each $r>0$ and every $\theta \in[0, \pi / 2]$, we have $u(x)=u(y)$, for every $x, y \in I(\theta, r)$. Thus $u$ is axially symmetric in $H$ and $u(x)=h(r, \theta)$, where $r=|x|$ and $\theta=\cos ^{-1} x_{n} / r$.

Proof. We again use reflection and comparison. The arguments are similar to those in Lemmas 2.2 and 2.5. Let $P_{1}, P_{2} \in I(\theta, r)$. Both $P_{1}$ and $P_{2}$ lie in the plane $\left\{x: x_{n}=r \cos \theta\right\}$. Set $\vec{e}=\left(P_{1}-P_{2}\right) /\left|P_{1}-P_{2}\right|$ and $P_{3}=\left(P_{1}+P_{2}\right) / 2$, then $\vec{e} \perp \overrightarrow{e_{n}}$ and $\vec{e} \perp P_{3}$. Define the hyperplane $Z(\vec{e})=\left\{x:\left\langle x-P_{3}, \vec{e}\right\rangle=\langle x, \vec{e}\rangle=0\right\}$. Note that $Z(\vec{e}) \ni O$. We tilt and translate $Z(\vec{e})$ as follows. Define $Z_{\varepsilon}(\vec{e})=\left\{x:\left\langle x, \vec{e}-\varepsilon \overrightarrow{e_{n}}\right\rangle=0\right\}$, where $\varepsilon>0$ is so small that $P_{1} \in Z_{\varepsilon}(\vec{e})^{+}=\left\{x:\left\langle x, \vec{e}-\varepsilon \overrightarrow{e_{n}}\right\rangle>0\right\}$. For $\delta>$ 0 , small, define the translated plane $Z_{\varepsilon, \delta}(\vec{e})=\left\{x+\delta\left(\vec{e}-\varepsilon \overrightarrow{e_{n}}\right): x \in Z_{\varepsilon}(\vec{e})\right\}$ and the half-space $Z_{\varepsilon, \delta}(\vec{e})^{+}$accordingly. For small $\delta>0, P_{1} \in Z_{\varepsilon, \delta}(\vec{e})^{+}$. We reflect the wedge $H \cap Z_{\varepsilon, \delta}(\vec{e})^{+}$about $Z_{\varepsilon, \delta}(\vec{e})$. We now apply comparison as was done in Lemma 2.5. For $x \in H \cap Z_{\varepsilon, \delta}(\vec{e})^{+}$, let $x^{f}(\varepsilon, \delta)$ be the reflection of $x$ about $Z_{\varepsilon, \delta}(\vec{e})$, and $u^{f}(x)=u\left(x^{f}(\varepsilon, \delta)\right)$. Comparison yields that $u^{f}(x) \leq u(x)$ in the wedge obtained by reflecting $H \cap Z_{\varepsilon, \delta}(\vec{e})^{+}$. Clearly, $u\left(P_{1}\right) \leq u\left(P_{1}^{f}(\varepsilon, \delta)\right)$. Letting $\varepsilon, \delta \rightarrow 0$, we see that $u\left(P_{1}\right) \leq u\left(P_{2}\right)$. Now replacing $P_{1}$ by $P_{2}$ and repeating the above argument we see $u\left(P_{1}\right) \geq u\left(P_{2}\right)$ and equality holds.

Remark 2.7. Using Lemma 2.5 and Lemma 2.6, it is clear that $u(x)=h(r, \theta)$ is decreasing in $\theta$. If $\rho=\left|x^{\prime}\right|$ then $u(x)=h(r, \theta)=g\left(\rho, x_{n}\right)$.

Remark 2.8. Adapting the arguments of Lemmas 2.5 and 2.6, it can be shown that $u(x)=u\left(\rho, x_{n}\right)$ is decreasing in $\rho$.

Lemma 2.9. Let $u$ be as in Theorem 1.1, $P \in H$ be a point on the $x_{n}$-axis and $\vec{e}$ be such that $\left\langle\vec{e}, \overrightarrow{e_{n}}\right\rangle>0$. Then for $t \geq 0, u(P+t \vec{e})$ is a decreasing function of $t$. In particular, $u=h(r, \theta)$ is decreasing in $r$. 
Proof. Select $P \in H$, then $P=(0, \eta)$ for some $\eta>0$. Set $H_{\eta}=\left\{x: x_{n}=\eta\right\}$ and $H_{\eta}^{+}=\left\{x: x_{n} \geq \eta\right\}$. For $x \in H_{\eta}^{+}$and $a>1$, let $x_{a}=a(x-P)+P$. Under this scaling

(i) $H_{\eta}^{+}$stays invariant,

(ii) $x$ and $x_{a}$ are collinear with $P$, and

(iii) if $x \in H_{\eta}$ then $x_{a} \in H_{\eta}$ and $\left|x^{\prime}\right|=|x-P|<\left|x_{a}^{\prime}\right|=a|x-P|$.

Now define $u_{a}(x)=u\left(x_{a}\right)$ in $H_{\eta}^{+}$. Then

(a) $u_{a}$ is $\infty$-harmonic in $H_{\eta}^{+}$,

(b) for $x \in H_{\eta}, u_{a}(x)=u\left(x_{a}\right) \leq u(x)$ (by Remarks 2.7 and 2.8), and

(c) by (A1) and (A2),

$$
\lim _{r \rightarrow \infty} \sup _{\{|x-P|=r\} \cap H_{\eta}^{+}} u(x)=\lim _{r \rightarrow \infty} \sup _{\{|x-P|=r\} \cap H_{\eta}^{+}} u_{a}(x)=0 .
$$

We may now adapt Lemma 2.2 to conclude that $u\left(x_{a}\right)=u_{a}(x) \leq u(x), x \in H_{\eta}^{+}$. This shows that $u(P+t \vec{e})$ is decreasing in $t$.

Now let $z \in H, t>1$ and $\vec{e}=z /|z|$. Let $L(z)$ be the ray $\{s z: s>0\}$. For small $\varepsilon>0$, set $P_{\varepsilon}=(0, \varepsilon), y=P_{\varepsilon}+|z| \vec{e}$, and $y_{t}=P_{\varepsilon}+t|z| \vec{e}$. Clearly, $|y-z|=\left|y_{t}-t z\right|=$ $\left|P_{\varepsilon}\right| \rightarrow 0$ as $\varepsilon \rightarrow 0$. From the previous argument it is clear that for every $\varepsilon>0$, $u\left(y_{t}\right) \leq u(y)$. Since $u$ is continuous in $H^{+}$, it follows that $u(t z) \leq u(z)$.

Remark 2.10. We may derive a lower bound for $u$ using Lemma 2.1 on $\partial B_{r}(O) \cap H$, $r>0$. Let $P=(0, r)$ and $Q=(r, \theta), 0<\theta<\pi / 2$. Fix $Q$ and let $L$ denote the circular arc joining $P$ to $Q$. Parameterizing $L$ by $\theta$, we see that $M(r) \leq(\sec \theta+\tan \theta) u(r, \theta)$. Working with the chord instead $[\sigma(t)=r(t \sin \theta, t \cos \theta+(1-t)), \delta(t)=r(1-t(1-$ $\cos \theta)), 0 \leq t \leq 1]$ we get $M(r) \leq u(r, \theta)(\sec \theta)^{\nu(\theta)}$, where $\nu(\theta)=\sqrt{2 /(1-\cos \theta)}$. Also if $P=\left(r_{1}, \theta\right)$ and $Q=\left(r_{2}, \theta\right), 0<r_{1}<r_{2}$, we may show by using Lemma 2.1 that $u\left(r_{1}, \theta\right) r_{1}^{\sec \theta} \leq u\left(r_{2}, \theta\right) r_{2}^{\sec \theta}$.

\section{Appendix}

We recall the example of a planar singular solution in $[2,7]$. In [2] this singular solution is expressed in terms of $r$ and $\theta$ which refer to the polar coordinates. This example is written as $u(x)=r^{-1 / 3} f(\theta)$ where

$$
\begin{aligned}
f(\theta) & =\frac{\cos t}{\left(1+2 \cos ^{2} t\right)^{2 / 3}}, \quad \theta=t-2 \arctan [(\tan t) / 2], \quad-\frac{\pi}{2} \leq t \leq \frac{\pi}{2} \\
\Delta_{\infty} u & =u_{r}^{2} u_{r r}+\frac{2 u_{r} u_{\theta} u_{r \theta}}{r^{2}}+\frac{u_{\theta}^{2} u_{\theta \theta}}{r^{4}}-\frac{u_{r} u_{\theta}^{2}}{r^{3}}=0 .
\end{aligned}
$$


Also $f(0)=1, f( \pm \pi / 2)=0, f^{\prime}(0)=0$, and $f(-\theta)=f(\theta)$. This solution is $C^{\infty}$ except at $\theta=0$ and it was verified in [7] (see the appendix of this work) that this is also a viscosity solution of (4) in the half-plane. We extend the planar example to $n>2$ as follows. Recall that in this work $\theta \in[0, \pi / 2]$, and refers to the opening of the cone with apex at $O$, and that every non-negative singular solution is axially symmetric. For $n>2$, define $r^{2}=\sum_{i=1}^{n} x_{i}^{2}, \cos \theta=x_{n} / r$, and set for $r>0,0 \leq \theta \leq \pi / 2$, $u(x)=h(r, \theta)=r^{-1 / 3} f(\theta)$. Now $u$ is defined in all of $H$. Routine calculations show that (4) holds in $0<\theta<\pi / 2$. Showing that $u$ is a viscosity solution in $H$ will largely be a repetition of the work in [7]. We provide details where slight differences occur. We take $\theta=0$. Let $\psi(x) \in C^{2}$ be such that $u(x)-\psi(x)$ has a maximum at $x^{1}=\left(0, r_{1}\right)$ on $x_{n}$-axis. Then as $x \rightarrow x^{1}$,

$$
\begin{aligned}
\frac{f(\theta)}{r^{1 / 3}}-\frac{1}{r_{1}^{1 / 3}} & \leq \psi(x)-\psi\left(x^{1}\right) \\
& =\sum_{i=1}^{n-1} \psi_{x_{i}}\left(x^{1}\right) x_{i}+\psi_{x_{n}}\left(x^{1}\right)\left(x_{n}-r_{1}\right)+O\left(\left|x-x^{1}\right|^{2}\right) .
\end{aligned}
$$

Taking $\theta=0$ and $x=(0, r)$, we see that $\psi_{x_{n}}\left(x^{1}\right)=-r_{1}^{-4 / 3} / 3$. For a fixed $i=1,2, \ldots, n-1$, set $x=s \overrightarrow{e_{i}}+x^{1}$. It is clear that for any $s \neq 0, s \psi_{x_{i}}+O\left(s^{2}\right) \geq$ $r^{-1 / 3} f(\theta)-r_{1}^{-1 / 3}$. It is easily seen that $\psi_{x_{i}}\left(x^{1}\right)=0$. Taking $x=\left(0, r_{1} \pm \delta\right), \delta>0$ and working with quadratic approximations, $\psi_{x_{n} x_{n}}\left(x^{1}\right) \geq 0$. Thus $\Delta_{\infty} \psi\left(x^{1}\right)=$ $\psi_{x_{n}}^{2}\left(x^{1}\right) \psi_{x_{n} x_{n}}\left(x^{1}\right) \geq 0$. Now let $u-\psi$ have a minimum at $x^{1}=\left(0, r_{1}\right)$. Then (5) holds with the inequality reversed. Once again $\psi_{x_{i}}\left(x^{1}\right)=0, i=1,2, \ldots, n-1$, and $\psi_{x_{n}}\left(x^{1}\right)=-r_{1}^{-4 / 3} / 3$. Using quadratic approximation with $x=s \overrightarrow{e_{i}}+x^{1}$ yields $\psi_{x_{i} x_{i}}\left(x^{1}\right) s^{2} \leq r^{-1 / 3} f(\theta)-r_{1}^{-1 / 3}$. Recalling that $f^{\prime \prime}(\theta) \rightarrow-\infty$ as $s \rightarrow 0$, we see that $\psi_{x_{i} x_{i}}\left(x^{1}\right) \leq-\infty$, contradicting that $\psi$ is $C^{2}$. Thus minimum occurs only when $\theta \neq 0$.

Acknowledgements. We are appreciative of the referee's suggestions which have improved the presentation and also thank Juan Manfredi for his comments on an earlier version.

\section{References}

[1] G. Aronsson, On certain singular solutions of the partial differential equation $u_{x}^{2} u_{x x}+2 u_{x} u_{y} u_{x y}+$ $u_{y}^{2} u_{y y}=0$, Manuscripta Math. 47 (1984), no. 1-3, 133-151.

[2] Construction of singular solutions to the p-harmonic equation and its limit equation for $p=\infty$, Manuscripta Math. 56 (1986), no. 2, 135-158.

[3] G. Aronsson, M. G. Crandall, and P. Juutinen, A tour of the theory of absolutely minimizing functions, Bull. Amer. Math. Soc. (N.S.) 41 (2004), no. 4, 439-505 (electronic).

[4] G. Barles and J. Busca, Existence and comparison results for fully nonlinear degenerate elliptic equations without zeroth-order term, Comm. Partial Differential Equations 26 (2001), no. 11-12, 2323-2337. 
[5] T. Bhattacharya, An elementary proof of the Harnack inequality for non-negative infinitysuperharmonic functions, Electron. J. Differential Equations (2001), no. 44, 8 pp. (electronic).

[6] - On the properties of $\infty$-harmonic functions and an application to capacitary convex rings, Electron. J. Differential Equations (2002), no. 101, 22 pp. (electronic).

[7] $\ldots$, On the behaviour of $\infty$-harmonic functions near isolated points, Nonlinear Anal. 58 (2004), no. 3-4, 333-349.

[8] - On the behaviour of $\infty$-harmonic functions on some special unbounded domains, Pacific J. Math., to appear.

[9] T. Bhattacharya, E. DiBenedetto, and J. Manfredi, Limits as $p \rightarrow \infty$ of $\Delta_{p} u_{p}=f$ and related extremal problems, Rend. Sem. Mat. Univ. Politec. Torino (1989), no. Special Issue, 15-68 (1991).

[10] M. G. Crandall, L. C. Evans, and R. F. Gariepy, Optimal Lipschitz extensions and the infinity Laplacian, Calc. Var. Partial Differential Equations 13 (2001), no. 2, 123-139.

[11] P. Lindqvist and J. J. Manfredi, The Harnack inequality for $\infty$-harmonic functions, Electron. J. Differential Equations (1995), no. 04, 5 pp. (electronic).

[12] J. J. Manfredi, Isolated singularities of p-harmonic functions in the plane, SIAM J. Math. Anal. 22 (1991), no. 2, 424-439 